\title{
BMJ Open Effect of vitamin D supplementation on inflammation: protocol for a systematic review
}

\author{
Aya Mousa, ${ }^{1}$ Marie Misso, ${ }^{1}$ Helena Teede, ${ }^{1,2}$ Robert Scragg, ${ }^{3}$ \\ Barbora de Courten ${ }^{1,2}$
}

To cite: Mousa A, Misso M, Teede $\mathrm{H}$, et al. Effect of vitamin D supplementation on inflammation: protocol for a systematic review. BMJ Open 2016;6:e010804. doi:10.1136/bmjopen-2015010804

- Prepublication history and additional material is available. To view please visit the journal (http://dx.doi.org/ 10.1136/bmjopen-2015010804).

Received 14 December 2015 Revised 10 February 2016 Accepted 15 February 2016

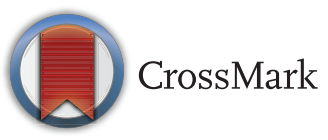

${ }^{1}$ Monash Centre for Health Research and Implementation (MCHRI), School of Public health and Preventive Medicine, Monash University, Melbourne, Victoria, Australia ${ }^{2}$ Diabetes and Vascular Medicine Unit, Monash Health, Melbourne, Victoria, Australia

${ }^{3}$ School of Population Health, University of Auckland, Auckland, New Zealand

Correspondence to Professor Barbora de Courten; barbora.decourten@ monash.edu

\section{ABSTRACT}

Introduction: The extraskeletal role of vitamin $D$ is being increasingly recognised. This has important clinical implications, as vitamin $\mathrm{D}$ deficiency has reached epidemic proportions worldwide. Vitamin D has proposed anti-inflammatory properties, yet the role of vitamin $D$ supplementation in reducing inflammation remains largely unknown. The purpose of this review is to investigate the impact of vitamin D supplementation on inflammation, and to identify relevant knowledge gaps in the field.

Methods and analysis: Medline, CINAHL, EMBASE and All EBM will be systematically searched for randomised controlled trials (RCTs) and systematic reviews of RCTs, comparing vitamin D supplementation with placebo, usual care or other pharmacological or non-pharmacological interventions. One reviewer will assess articles for eligibility according to prespecified selection criteria, after which 2 independent reviewers will perform data extraction and quality appraisal. Meta-analyses will be conducted where appropriate.

Ethics and dissemination: Formal ethical approval is not required as no primary data is collected. This systematic review will identify potential clinical implications of vitamin $\mathrm{D}$ deficiency and supplementation, and will be disseminated through a peer-reviewed publication and at conference meetings, to inform future research on the efficacy of vitamin $D$ supplementation for inflammation and inflammatory diseases.

PROSPERO registration number: CRD42016037104.

\section{INTRODUCTION}

Vitamin D has traditionally been known for its role in regulating calcium and phosphorus for the healthy mineralisation of bone. Recent evidence has broadened interest to the role of vitamin D in extraskeletal functions, including in inflammation and immunoregulation. ${ }^{12}$ The functions of vitamin $\mathrm{D}$ in inflammation are of increasing interest, and vitamin $\mathrm{D}$ deficiency has been implicated

\section{Strengths and limitations of this study}

- Novel and relevant areas of research which will guide future research in this field.

- Protocol is for the first systematic review to investigate vitamin $D$ and inflammation across all population groups and disease and/or nondisease states.

- Employs rigorous international gold-standard methodology and comprehensive search strategy.

- Limited in that the systematic review will include only published data.

in the pathophysiology of various inflammatory diseases including Crohn's disease and rheumatoid arthritis, as well as in conditions associated with chronic low-grade inflammation, such as obesity, insulin resistance, type 2 diabetes and cardiovascular disease. ${ }^{3}$

The interaction between vitamin $\mathrm{D}$ and inflammation may have clinical implications, as vitamin $\mathrm{D}$ deficiency remains prevalent worldwide and is increasing as a result of sedentary indoor lifestyles and the use of sunscreen and protective clothing to reduce the risk of skin cancer. ${ }^{4}$ At present, there is no universal consensus on optimal levels of vitamin $\mathrm{D}$, but most experts agree that plasma 25-hydroxyvitamin D levels $<50 \mathrm{nmol} / \mathrm{L}$ would be considered deficient. ${ }^{5}$ It is therefore concerning that $20-60 \%$ of the UK and 10 $40 \%$ of the US population have vitamin D levels $<50 \mathrm{nmol} / \mathrm{L}$. ${ }^{6}$ Despite the sunny climate in Australia, vitamin D deficiency is prevalent in $50 \%$ of women and $31 \%$ of men.

Vitamin D can be obtained from dietary sources or supplements in the form of cholecalciferol or ergocalciferol, although it is primarily derived via conversion of 7-dehydrocholesterol in the skin consequent to exposure to ultraviolet $\mathrm{B}$ radiation. ${ }^{8}$ However, recommendations to prevent skin cancer by reducing sun exposure have made it difficult to obtain adequate vitamin D 
through sun exposure, and limited foods have naturally high vitamin D levels, or are vitamin D fortified. ${ }^{4}$

Following ingestion or cutaneous synthesis, vitamin D is hydroxylated by the liver into 25-hydroxyvitamin $\mathrm{D}_{2}$ or $\mathrm{D}_{3}$ (25OHD) and is then converted to its biologically active form (1,25-dihydroxyvitamin $\mathrm{D}_{2} / \mathrm{D}_{3}$ or $1,25 \mathrm{OHD}$, for short) via a second hydroxylation in the kidney by 25OHD-1 $\alpha$-hydroxylase (CYP27B1). ${ }^{9} 1,25 \mathrm{OHD}$ functions as a steroid hormone and binds to a nuclear vitamin D receptor (VDR) which has recently been found in nearly all tissue cells, including most inflammatory cells, with particularly high VDR levels in dendritic cells, macrophages and $\mathrm{T}$ and $\mathrm{B}$ lymphocytes, thus supporting the notion that vitamin D may have a role in inflammatory and immune responses. ${ }^{10}$

Several studies have attempted to delineate the effects of vitamin $\mathrm{D}$ on inflammatory cells and processes. In vitro, vitamin $\mathrm{D}$ has been shown to promote monocyte differentiation to macrophages, preventing them from releasing inflammatory cytokines and reducing their ability to present antigens to lymphocytes by inhibiting cell surface expression of the class 2 major histocompatibility complex (MHC-II) molecule. ${ }^{2}$ Vitamin D also suppresses the proliferation and stimulatory abilities of $\mathrm{T}$ cells and monocytes, and downregulates proinflammatory cytokines, including $\mathrm{C}$ reactive protein (CRP), tumour necrosis factor $\alpha(\mathrm{TNF} \alpha)$, interleukin (IL) 6 , IL-1 and IL-8, while upregulating anti-inflammatory cytokines such as IL-10. ${ }^{2}$ In vitro data has also shown associations between absence of the VDR and increased nuclear factor $\kappa \mathrm{B}(\mathrm{NF \kappa B})$ activity, a transcription factor with a key role in immunomodulation, and in the pathophysiology of several inflammatory diseases and chronic inflammatory states. ${ }^{11}$ The reverse was also evident, where vitamin $\mathrm{D}$ was shown to prevent NFкB translocation and weaken its activity. ${ }^{11}$

In vivo studies using animal models have shown that supplementation with $1,25 \mathrm{OHD}$ prevented the development of inflammatory arthritis, type 1 diabetes and autoimmune encephalomyelitis and thyroiditis. ${ }^{12-15}$ Administration of $1,25 \mathrm{OHD}$ to non-obese, non-diabetic mice also modulated chemokine and cytokine expression and prevented diabetes. ${ }^{16}$ Conversely, VDR knockout mice developed severe diarrhoea and rectal bleeding, suggesting that vitamin D deficiency may compromise the integrity of the intestinal mucosal barrier, thereby increasing susceptibility to mucosal damage and development of inflammatory bowel disease. ${ }^{17}$

Human observational studies have frequently reported that higher vitamin $\mathrm{D}$ levels were associated with lower inflammatory markers including CRP, IL-6 and $\mathrm{TNF} \alpha$ in healthy populations, ${ }^{18-20}$ and in those with proinflammatory conditions, such as diabetes, arteriosclerosis and inflammatory polyarthritis. ${ }^{3}$ Vitamin $\mathrm{D}$ has also been associated with adipocytokines, with studies showing inverse correlations between vitamin D and leptin, ${ }^{21}{ }^{22}$ and positive correlations between vitamin $\mathrm{D}$ and adiponectin. ${ }^{22} 23$ This latter action of vitamin $\mathrm{D}$ is thought to occur partly via down-regulating IL-6 since IL-6 has been shown to inhibit adiponectin gene expression. ${ }^{23} 24$

It is biologically plausible that sufficient vitamin D levels may be important in modulating inflammatory processes, and this seems to be supported by various experimental and observational studies. ${ }^{1}{ }^{2}$ However, observational studies are limited given their high risk of bias and inability to establish causation or directionality. Moreover, randomised controlled trials (RCTs) and systematic reviews of RCTs to date have focused on specific population groups, such as children, ${ }^{25}{ }^{26}$ or inflammatory conditions, such as obesity, inflammatory bowel disease and chronic obstructive pulmonary disease. ${ }^{27-30}$ Thus, there remains a gap in knowledge of the effects of vitamin D supplementation on inflammatory markers or states across the wider general population. This systematic review, therefore, aims to address this gap by identifying and comprehensively synthesising evidence evaluating the effect of vitamin D supplementation on inflammation in all population groups.

\section{SYSTEMATIC REVIEW QUESTION}

- Is vitamin D supplementation effective for treatment of inflammation (versus placebo or usual care)?

- Is vitamin D supplementation better than other nonpharmacological or pharmacological interventions for treatment of inflammation?

\section{METHODS/DESIGN}

Rigorous international gold-standard methodology will be adopted in this review, ${ }^{31} 32$ and will conform to the reporting standards of the Preferred Reporting Items for Systematic Reviews and Meta-analyses (PRISMA). This systematic review has been registered on PROSPERO under the identification code: CRD42016037104.

\section{Eligibility criteria}

Selection criteria using the PICO (Population, Intervention, Comparison, Outcomes) framework in table 1 established a priori will be used to determine eligibility of articles.

\section{SEARCH STRATEGY}

A systemic search will be developed using relevant search terms (see online supplementary file 1) in accordance with the selection criteria (table 1), and the following electronic databases will be used:

- MEDLINE via OVID;

- MEDLINE in-process and other non-indexed citations via OVID;

- CINAHL;

- EMBASE via OVID;

- All Evidence Based Medicine (EBM) Reviews via OVID incorporating: The Cochrane Library; Cochrane Database of Systematic Reviews (Cochrane 
Table 1 PICO for study inclusion

\begin{tabular}{|c|c|c|c|}
\hline Participants (P) & Intervention (I) & Comparison (C) & Outcomes (0) \\
\hline \multicolumn{4}{|l|}{ Inclusion criteria } \\
\hline $\begin{array}{l}\text { Males and females of } \\
\text { any age, ethnicity, } \\
\text { socioeconomic status, } \\
\text { geographic area, } \\
\text { comorbidity or pregnancy } \\
\text { status }\end{array}$ & $\begin{array}{l}\text { Vitamin } \mathrm{D}_{2} \text { and/or vitamin } \mathrm{D}_{3} \\
\text { supplementation, administered } \\
\text { in any form (intravenous, } \\
\text { intramuscular, or oral), alone } \\
\text { or combined with other } \\
\text { intervention/s, of any dosage, } \\
\text { and for any duration }\end{array}$ & $\begin{array}{l}\text { Placebo or usual care. Any } \\
\text { other non-pharmacological } \\
\text { interventions or } \\
\text { pharmacological interventions }\end{array}$ & $\begin{array}{l}\text { Inflammatory biomarkers } \\
\text { including, but not limited to: } \\
\text { all interleukins, all TNF } \alpha \text {, } \\
\text { TGF- } \beta 1 \text {, hs/CRP, MCP-1, } \\
\text { ADMA, PAI-1, SAA, IFN } \gamma \text {, } \\
\text { NFאB, MIF, ICAMs, } \\
\text { fibrinogen, MMPs, } \\
\text { adipocytokines: leptin, } \\
\text { resistin, visfatin, adiponectin, } \\
\text { omentin }\end{array}$ \\
\hline \multicolumn{4}{|l|}{ Exclusion criteria } \\
\hline None & \multicolumn{3}{|l|}{$\begin{array}{l}\text { Studies without vitamin D } \\
\text { supplementation }\end{array}$} \\
\hline Study type & \multicolumn{3}{|c|}{ Systematic reviews of RCTs and RCTs } \\
\hline Language & \multicolumn{3}{|l|}{ No limit } \\
\hline Year of publication & \multicolumn{3}{|l|}{ No limit } \\
\hline
\end{tabular}

Reviews); Database of Abstracts of Reviews of Effects (Other Reviews); Cochrane Central Register of Controlled Trials (Clinical Trials); Cochrane Database of Methodology Reviews (Methods Reviews); The Cochrane Methodology Register (Methods Studies); Health Technology Assessment Database (Technology Assessments); NHS Economic Evaluation Database (Economic Evaluations); and ACP Journal Club.

Bibliographies of relevant studies identified by the search strategy and relevant reviews/meta-analyses will also be searched for identification of additional studies. Corresponding authors of any included trials where required data are not presented will be contacted to provide deidentified data (aggregated effect measures) for the purpose of meta-analysis, if deemed appropriate. To identify unpublished studies we will also search the National Institute of Health Clinical Trials Register (https://clinicaltrials.gov/) and the Australian New Zealand Clinical Trials registries (https://www.anzctr. org.au).

\section{Inclusion of studies}

To determine the literature to be assessed further, one reviewer will scan the titles, abstract sections and keywords of every record retrieved by the search strategy using the selection criteria described in table 1 , and in consultation with a second reviewer. Disagreement between reviewers about whether a study meets the inclusion criteria will be resolved by discussion. Where consensus is not reached, a third reviewer will be consulted to reach a decision. A pilot test of the selection criteria will be conducted on 3-5 articles in order to refine and clarify the criteria prior to screening.
Full articles will be retrieved for further assessment if the information given suggests that the study meets the selection criteria. Where there is any doubt regarding eligibility from information provided in the title and abstract, the full article will be retrieved for clarification. Studies excluded based on full text will be tabulated with the reason/s for their exclusion.

\section{Quality appraisal of the evidence}

Methodological quality, in terms of risk of bias, of the included studies will be assessed at the study level by two independent reviewers using criteria developed a priori, as outlined in the Monash Centre for Health Research and Implementation critical appraisal template. ${ }^{33}$ Individual quality items will be investigated using a descriptive component approach that includes items such as conflict of interest of authors, presence of prespecified selection criteria, methods of randomisation and allocation of participants to study groups, blinding of participants, carers, investigators or outcome assessors, methods of outcome assessment and reporting, and statistical issues, such as powering and methods of data analysis (see online supplementary file 2). Any disagreement or uncertainty will be resolved by discussion among review authors to reach a consensus. Using this approach, each study will be allocated a risk of bias rating. ${ }^{33}$

\section{Data extraction}

Data will be extracted from all included studies by two independent reviewers using a specifically developed data extraction form in line with the selection criteria and outcomes of interest (table 1). Both reviewers will 
check all computed data entries for meta-analyses, if applicable.

Extracted data will include mean values and SDs of the outcomes, and their CIs, point estimates and measures of variability, frequency counts for dichotomous variables, number of participants, intention-to-treat analysis, and validity results.

Outcomes may be reported as continuous measures of these inflammatory biomarkers, and in such cases, the mean or standardised mean difference along with their SDs and CIs will be used to measure the effects (see online supplementary file 2). Eligible outcomes may also be dichotomous and, as such, will include relative measures of inflammation risk (risk ratio or $\mathrm{OR}$ along with CIs), or absolute numbers of patients experiencing at least one episode of inflammation.

\section{Grading the body of evidence}

The quality of the evidence for the effects of vitamin D supplementation on inflammatory markers and diseases will be assessed as high, moderate, low or very low using the GRADE approach. ${ }^{34}$ Quality of evidence will be graded by two independent reviewers based on risk of bias, imprecision, heterogeneity, indirectness and suspicion of publication bias. Quality will be reported at the study level, and where appropriate, at the outcome level, in line with PRISMA guidelines. Disagreements will be resolved by discussion, and where consensus cannot be reached, a third reviewer will be consulted. Interpretation of the grading scores is presented in table 2.

\section{DATA ANALYSIS AND SYNTHESIS \\ Planned analyses}

Data will be presented in summary tables and in narrative form to describe the populations, interventions and outcomes of the included studies. Where data is

$\begin{aligned} & \text { Table } 2 \text { Grading the quality of the evidence (adapted } \\ & \text { from GRADE Working Group, 2004) }\end{aligned}$
$\begin{array}{ll}\text { Strength of } \\ \text { evidence }\end{array}$
$\begin{array}{ll}\text { Interpretation } \\ \text { High quality }\end{array}$
$\begin{aligned} & \text { Very confident in the estimate of the } \\ & \text { effect, and further research is very } \\ & \text { unlikely to change our confidence. }\end{aligned}$
$\begin{aligned} & \text { Moderately confident in the estimate of } \\ & \text { the effect, but further research may } \\ & \text { have an important impact on our } \\ & \text { confidence and may change the } \\ & \text { estimate. } \\ & \text { Somewhat confident in the estimate of } \\ & \text { the effect, but further research is very } \\ & \text { likely to have an important impact on } \\ & \text { our confidence and will likely change } \\ & \text { the estimate. } \\ & \text { Very little confidence in the estimate of } \\ & \text { the effect as it is very uncertain. }\end{aligned}$
Very low quality

available, or could be imputed or obtained from authors, between-group differences will be presented, and relative differences in outcomes will be assessed. Log transformation will be conducted where necessary. Aggregated effect measures will be used for meta-analyses where appropriate, when data are derived from clinically homogeneous groups (where participants, interventions and outcome measures are sufficiently similar), using a random effects model in Review Manager 5.3.5. Dichotomous outcomes will be presented as relative risks with $95 \%$ CIs, while continuous outcomes will be presented as weighted mean differences with $95 \%$ CIs. A $p$ value of $<0.05$ will indicate statistical significance.

Statistical heterogeneity will be assessed using the $\mathrm{I}^{2}$ test, where $\mathrm{I}^{2}$ values over $50 \%$ indicate moderate to high heterogeneity. Descriptive analyses will be conducted for those studies which are deemed clinically heterogeneous, or present insufficient data for pooling.

\section{Subgroup and sensitivity analyses}

Subgroup analyses, or if applicable, metaregression analyses will be performed for factors presumed to cause variations in outcomes, and may include age, body mass index, dosage regimen, deficiency of participants at baseline, participant disease status and study duration.

Sensitivity analysis will be conducted if deemed appropriate, and factors to be included will be determined during the review process. Heterogeneity $\mathrm{I}^{2}>50 \%$ will be explored through sensitivity analysis using risk of bias. For meta-analyses of more than 10 studies, funnel plots will be used to determine small study effects and potential publication bias.

\section{DISCUSSION}

Although inflammatory processes are crucial for the human host defence against infectious agents and injury, prolonged systemic inflammation contributes to the pathophysiology of many chronic diseases. ${ }^{1}$ Moreover, there is limited knowledge on the potential of naturally occurring nutrients, such as vitamin $\mathrm{D}$, in improving these inflammatory states and conditions.

To the best of our knowledge, this will be the first comprehensive systematic review investigating the effectiveness of vitamin D supplementation in improving inflammation in all populations, as previous reviews have been limited to investigating specific inflammatory conditions or population groups. ${ }^{26} 28{ }^{30} \mathrm{In}$ addition, this review will include trials investigating inflammation independently, and/or as a risk factor in the pathophysiology of cardiometabolic diseases such as type 2 diabetes, heart disease or chronic inflammatory conditions such as Crohn's disease and rheumatoid arthritis. There is also a lack of sound evidence to justify biological or sociological differences between population subgroups, hence, the additional benefit of including populations of all ages and backgrounds in this review. 
Our systematic review will use rigorous methodology, prespecified criteria and predetermined outcomes in order to comprehensively examine and synthesise the literature and assess the effects of vitamin D supplementation on inflammatory markers and diseases in a variety of populations.

Although outside of our control, this systematic review is limited by its reliance on published data, and thus, publication bias cannot be ruled out as there may be unpublished findings that are unaccounted for.

This systematic review will investigate the impact of vitamin D supplementation on inflammation, and identify knowledge gaps to inform future research in the field. If vitamin D supplementation improves inflammation on systematic review and meta-analyses, we will generate level 1 evidence of efficacy with important implications for clinical practice.

Acknowledgements AM is a recipient of the Australian Postgraduate Award Scholarship provided by Monash University. BdC is supported by the Australian National Health and Medical Research Council.

Contributors AM designed and wrote the review protocol, and will coordinate the review process. MM contributed to the design of the search strategy and statistical methods, revised the manuscript, and will contribute to data collection and analysis. HT and RS revised and edited the manuscript. BdC determined the scope of the review, revised and edited the manuscript, and is the guarantor.

Competing interests None declared.

Provenance and peer review Not commissioned; externally peer reviewed.

Open Access This is an Open Access article distributed in accordance with the Creative Commons Attribution Non Commercial (CC BY-NC 4.0) license, which permits others to distribute, remix, adapt, build upon this work noncommercially, and license their derivative works on different terms, provided the original work is properly cited and the use is non-commercial. See: http:// creativecommons.org/licenses/by-nc/4.0/

\section{REFERENCES}

1. Cannell JJ, Grant WB, Holick MF. Vitamin D and inflammation. Dermatoendocrinol 2014;6:e983401.

2. Guillot X, Semerano L, Saidenberg-Kermanac'h N, et al. Vitamin D and inflammation. Joint Bone Spine 2010;77:552-7.

3. Zittermann $A$. Vitamin $D$ in preventive medicine: are we ignoring the evidence? Br J Nutr 2003;89:552-72.

4. Holick MF. Vitamin D status: measurement, interpretation, and clinical application. Ann Epidemiol 2009;19:73-8.

5. Zittermann A. Vitamin D and cardiovascular disease. Anticancer Res 2014;34:4641-8.

6. Prentice A. Vitamin D deficiency: a global perspective. Nutr Rev 2008;66(10 Suppl 2):S153-64.

7. Gagnon C, Lu ZX, Magliano DJ, et al. Serum 25-hydroxyvitamin D, calcium intake, and risk of type 2 diabetes after 5 years: results from a national, population-based prospective study (the Australian Diabetes, Obesity and Lifestyle study). Diabetes Care 2011;34:1133-8.

8. Wang C. Role of vitamin d in cardiometabolic diseases. J Diabetes Res 2013;2013:243934.

9. Norman AW, Bouillon R. Vitamin D nutritional policy needs a vision for the future. Exp Biol Med (Maywood) 2010;235:1034-45.

10. Veldman CM, Cantorna MT, Deluca HF. Expression of 1,25-dihydroxyvitamin $D(3)$ receptor in the immune system. Arch Biochem Biophys 2000;374:334-8.

11. Harant H, Andrew PJ, Reddy GS, et al. 1 Alpha,25-dihydroxyvitamin D3 and a variety of its natural metabolites transcriptionally repress nuclear-factor-kappa B-mediated interleukin-8 gene expression. Eur J Biochem 1997;250:63-71.

12. Cantorna MT, Hayes CE, Deluca HF. 1,25-Dihydroxyvitamin D3 reversibly blocks the progression of relapsing encephalomyelitis, a model of multiple sclerosis. Proc Natl Acad Sci USA 1996;93:7861-4.

13. Cantorna MT, Hayes CE, Deluca HF. 1,25-Dihydroxycholecalciferol inhibits the progression of arthritis in murine models of human arthritis. J Nutr 1998;128:68-72.

14. Lemire JM, Archer DC. 1,25-Dihydroxyvitamin D3 prevents the in vivo induction of murine experimental autoimmune encephalomyelitis. J Clin Invest 1991;87:1103-7.

15. Zella JB, Mccary LC, Deluca HF. Oral administration of 1,25-dihydroxyvitamin D3 completely protects NOD mice from insulin-dependent diabetes mellitus. Arch Biochem Biophys 2003;417:77-80.

16. Gysemans CA, Cardozo AK, Callewaert $\mathrm{H}$, et al. 1,25-Dihydroxyvitamin D3 modulates expression of chemokines and cytokines in pancreatic islets: implications for prevention of diabetes in nonobese diabetic mice. Endocrinology 2005;146:1956-64.

17. Kong J, Zhang Z, Musch MW, et al. Novel role of the vitamin D receptor in maintaining the integrity of the intestinal mucosal barrier. Am J Physiol Gastrointest Liver Physiol 2008;294:G208-16.

18. De Vita F, Lauretani F, Bauer J, et al. Relationship between vitamin $\mathrm{D}$ and inflammatory markers in older individuals. Age (Dordr) 2014;36:9694.

19. Laird $\mathrm{E}$, McNulty $\mathrm{H}$, Ward M, et al. Vitamin D deficiency is associated with inflammation in older Irish adults. J Clin Endocrinol Metab 2014;99:1807-15.

20. Liefaard MC, Ligthart S, Vitezova A, et al. Vitamin D and C-reactive protein: a Mendelian randomization study. PLOS ONE 2015;10: e0131740

21. Fantuzzi G, Faggioni R. Leptin in the regulation of immunity, inflammation, and hematopoiesis. J Leukoc Biol 2000;68:437-46.

22. Stokic E, Kupusinac A, Tomic-Naglic D, et al. Vitamin D and dysfunctional adipose tissue in obesity. Angiology 2015;66:613-18.

23. Vaidya A, Williams JS, Forman JP. The independent association between 25 -hydroxyvitamin $\mathrm{D}$ and adiponectin and its relation with BMI in two large cohorts: the NHS and the HPFS. Obesity (Silver Spring) 2012;20:186-91.

24. Beilfuss J, Berg V, Sneve M, et al. Effects of a 1-year supplementation with cholecalciferol on interleukin-6, tumor necrosis factor-alpha and insulin resistance in overweight and obese subjects. Cytokine 2012;60:870-4.

25. Holmes EA, Xiang F, Lucas RM. Variation in incidence of pediatric Crohn's disease in relation to latitude and ambient ultraviolet radiation: a systematic review and analysis. Inflamm Bowel Dis 2015;21:809-17.

26. Riverin BD, Maguire JL, Li P. Vitamin D supplementation for childhood asthma: a systematic review and meta-analysis. PLOS ONE 2015;10:e0136841.

27. Chandler PD, Wang L, Zhang $X$, et al. Effect of vitamin $D$ supplementation alone or with calcium on adiposity measures: a systematic review and meta-analysis of randomized controlled trials. Nutr Rev 2015;73:577-93.

28. Del Pinto R, Pietropaoli D, Chandar AK, et al. Association between inflammatory bowel disease and vitamin $D$ deficiency: a systematic review and meta-analysis. Inflamm Bowel Dis 2015;21:2708-17.

29. Lu C, Yang J, Yu W, et al. Association between 25(OH)D level, ultraviolet exposure, geographical location, and inflammatory bowel disease activity: a systematic review and meta-analysis. PLOS ONE 2015;10:e0132036.

30. Zhu B, Zhu B, Xiao C, et al. Vitamin D deficiency is associated with the severity of COPD: a systematic review and meta-analysis. Int $J$ Chron Obstruct Pulmon Dis 2015;10:1907-16.

31. Julian SG, Higgins PT, eds. Cochrane handbook for systematic reviews of interventions. Chichester, UK: The Cochrane Library, John Wiley \& Sons, 2006.

32. Finding the Evidence. Centre for evidence based medicine. Oxford: University of Oxford, 2014.

33. MCHRI, Evidence Synthesis Program templates for critical appraisal and risk of bias (adapted from Critical Appraisal Templates, Centre for Clinical Effectiveness, Southern Health, Melbourne, 2010). Monash University and Monash Health, 2013.

34. Group GW. Grading quality of evidence and strength of recommendations. BMJ 2004;328:1490. 